\title{
EDUCACIÓN MUSICAL EN PORTUGAL DURANTE LA PRIMERA REPÚBLICA (1910-1926)*
}

\section{Music Education in Portugal during the First Republic (1910- 1926)}

\section{Bruno Borralhinho\&}

Fecha de recepción: 02/12/2016 • Fecha de aceptación: 05/12/ 2017

Resumen. El movimiento republicano eclosionó en Portugal en las últimas décadas del siglo XIX, seguramente impulsado por una asimilación natural - aunque tardía en comparación con otros países europeos- de los valores que sucedieron al Antiguo Régimen, pero sobretodo en un contexto de desgaste del bipartidismo de la monarquía constitucional portuguesa. La corona intenta contener la inestabilidad política incluso con soluciones gubernamentales autoritarias, pero el regicidio de 1908 en el que fallecen el rey D. Carlos y el príncipe heredero Luís Filipe, acaban por ser un presagio a la caída de la monarquía dos años después. El día 5 de octubre de 1910, como de cierto modo se esperaba, triunfa finalmente la revolución republicana y el país registra una serie de transformaciones sociales y políticas relevantes, aunque en muchos casos la revolución no representó un corte súbito con el pasado, pero sí la confirmación de una fase de transición y transformación de la sociedad portuguesa en el ámbito cultural y artístico. El sector musical - este artículo se refiere exclusivamente a la música clásica, comúnmente conocida por música vocal e instrumental occidental, excluyendo sectores paralelos como la música electrónica o el jazz, por ejemplo- no fue ajeno a esas transformaciones, y este texto investiga algunos casos paradigmáticos del clima novedoso y efervescente de la Primera República, pero también reveladores de una cierta inestabilidad e incoherencia de un tiempo más distinguido por sus expectativas que por sus logros.

Palabras clave: Educación musical; Música; Portugal; Primera República.

\footnotetext{
* Artículo basado en una sección temática de la tesis doctoral del autor en el Departamento Humanidades (Historia, Geografía y Artes) de la Universidad Carlos III (Madrid) con el título Poder y Música Clásica en el Portugal del Siglo XX.

\& Escuela de Doctorado. Departamento de Humanidades (Historia, Geografía y Arte). Universidad Carlos III de Madrid. Calle Madrid, 126. 28903 Getafe (Madrid). España. bruno_borralhinho@hotmail.com
}

Cómo citar este artículo: Borralhinho, Bruno. «Educación musical en Portugal durante la Primera República (1910-1926)». Historia y Memoria de la Educación 8 (2018): 597-617. 
Abstract. The republican movement erupted in Portugal in the last decades of the nineteenth century, probably driven by a natural assimilation -although late in comparison with other European countries-of the values that followed the Ancien Régime, but above all by the exhaustion of the bipartisan system of the Portuguese constitutional monarchy. The crown tried to contain the political instability with authoritarian governmental solutions, but the regicide of 1908 in which the king D. Carlos and the crown prince Luis Filipe were murdered ended up being an omen of the fall of the monarchy two years later. On October 5, 1910 the republican revolution triumphed, not surprisingly, and the country experienced a series of important social and political transformations. In many cases the revolution did not represent a sudden break with the past but rather the confirmation of a new phase of transition and transformation of Portuguese society, especially in the cultural and artistic field. The music sector - this article refers exclusively to classical music, commonly known as western vocal and instrumental music, excluding parallel sectors such as electronic music or jazz, for example - was not alien to these transformations. This text investigates some paradigmatic cases of the new and effervescent climate of the First Republic, revealing a certain instability and incoherence of a period more distinguished by its expectations than by its achievements.

Keywords: Music education; Music; Portugal; First Republic.

\section{INTRODUCCIÓN}

Una de las brechas más significativas en el panorama de la historia de la enseñanza musical en Portugal se refiere al período posterior a la revolución republicana del 1910. Este artículo surge en el contexto de una investigación más amplia sobre la relación entre la música y el poder de la Primera República portuguesa y pretende compensar la inexistencia de otros trabajos explícitamente sobre esta correspondencia.

Con la recopilación y revisión de literatura dispersa sobre subtemas tan diversos como, por ejemplo, la financiación, la elaboración de programas curriculares, el reclutamiento y selección de profesores o la relación entre instituciones públicas y privadas, se pretende dar consistencia a un hilo argumentativo - apoyado por fuentes primarias, fundamentalmente legislación- más consistente sobre la correlación entre el medio musical y las políticas republicanas. 


\section{EDUCACIÓN: UNA PRIORIDAD DEL IDEAL REPUBLICANO}

La tasa de analfabetismo de $75,1 \%$ de la población sofocaba a la sociedad portuguesa, a diferencia de Inglaterra o Alemania donde los porcentajes no superaban respectivamente $0,1 \%$ y $0,51 \%$. [... No es de extrañar que pocos meses después de la proclamación de la República se cambiasen las pautas del sistema educativo, los planes de estudio y programas para tratar de acortar distancias entre Portugal y la Europa culta. ${ }^{1}$

Los números y, sobre todo, el contraste con otras naciones europeas fueron la motivación y justificación definitivas para la urgencia en la implementación de los ideales republicanos. El país era mayoritariamente rural e incluso en las grandes ciudades la escuela no participaba de las costumbres o prioridades de las personas, más preocupadas por la supervivencia y la contribución de sus hijos para el sustento de sus familias, a través de su entrada prematura en el mercado laboral. El combate contra el analfabetismo - apostando también por la expansión de la educación popular - y la ejecución de diversas reformas estructurales con el objetivo de rehabilitar y mejorar la enseñanza en general, fueron algunas de las medidas más significativas del poder republicano.

La «dimensión política» de la educación —al igual que la «dimensión nacionalista y patriótica»_ era asumidas también como una de las prioridades de los políticos republicanos, que defendían que «la escuela debe ayudar a formar al nuevo ciudadano, republicano, democrático». ${ }^{2}$

[...] algunos de los más carismáticos líderes republicanos como Afonso Costa e António José Almeida defendían, respectivamente, la instrucción como grande cruzada de la República o que la «consolidación y gentrificación sólo se pueden llevar a cabo abriendo escuelas, esparciendo la educación, iluminando y rescatando a la juventud». ${ }^{3}$

\footnotetext{
${ }^{1}$ Ana Teresa Peixinho y Clara Almeida Santos (coords.), 1911-2011: Comunicação e Educação Republicanas (Coimbra: Universidade de Coimbra, 2011), 330.

${ }^{2}$ Maria José Conde Artiaga Barreiros, «A disciplina de Canto Coral no período do Estado Novo» (Tesis de Máster, Universidade Nova de Lisboa, Faculdade de Ciências Sociais e Humanas, 1999), 28.

${ }^{3}$ Peixinho y Santos: 1911-2011, 330. La cita final corresponde a un texto de Almeida de 1911.
} 
En 1911, se decretan medidas importantes como la creación de las universidades superiores en Lisboa y Oporto o la introducción de una asignatura de Educación Cívica en la educación primaria, en sustitución de la asignatura de Educación Moral. En este último caso, el objetivo era claro: «el proyecto de secularización de la sociedad» como «marco del discurso y de la práctica política republicana». ${ }^{4} \mathrm{El}$ poder asume, por lo tanto, la responsabilidad de reformar el sector y se inspira en ideales progresistas, «concepciones iluministas» y en la «filosofía positivista y en la propia dinámica generada por la Revolución Francesa de finales del siglo XVIII, [...] entendida como la esencia de la sociedad y el único medio capaz de superar el oscurantismo de una sociedad desigual que parecía parada en el tiempo».5

El déficit presupuestario era crónico y la deuda pública impedía u obstaculizaba la aplicación de medidas estructurales que ayudasen a mejorar la situación en un país con profundos desequilibrios sociales y culturales. ${ }^{6}$

Aunque la literatura sobre la época pueda vislumbrar que los resultados fuesen menores y más modestos que las intenciones o los proyectos republicanos, la historia reconoce el protagonismo que la educación mereció durante la Primera República, así como la importancia que esta circunstancia tuvo para las generaciones futuras.

\section{MÚSICA: AUTONOMÍA Y EXCLUSIVIDAD}

La música fue el ámbito que «centró mayoritariamente la atención de las autoridades y los actores educativos». ${ }^{7}$ Asimismo, y comparativamente con otros ramos de intervención del Estado portugués en el ámbito de la enseñanza, pudo disfrutar de un estatuto de exclusividad, notorio en varios aspectos y momentos. El mejor ejemplo fue el Conservatório Nacio-

\footnotetext{
${ }^{4}$ Joaquim Pintassilgo, «A utopia demopédica da República», en Viva a República! 1910-2010, coord. Luís Farinha (Lisboa: Comissão Nacional para as Comemorações da República, Imprensa Nacional Casa da Moeda, 2010), 139.

${ }^{5}$ Peixinho y Santos: 1911-2011, 9.

${ }^{6}$ Peixinho y Santos: 1911-2011, 330.

${ }^{7}$ Domingos Fernandes, Jorge Ramos do Ó y Mário Boto Ferreira (coord.), Estudo de Avaliação do ensino artístico (Lisboa: Direcção Geral de Formação Vocacional do Ministério da Educação e Faculdade de Psicologia e de Ciências da Educação da Universidade de Lisboa, 2007), 238.
} 
nal $(\mathrm{CN}),{ }^{8}$ aglutinador durante más de un siglo de toda la educación musical bajo responsabilidad gubernamental. ${ }^{9}$ La historia de esta institución educativa revela como el poder central «ha concedido y legitimado formatos organizativos absolutamente excepcionales en el cuadro de educación pública» en Portugal, y que «el fortísimo impulso centralizador del edificio educativo portugués [...] parece encontrar aquí su única negación». ${ }^{10}$

La Primera República heredó, en pleno siglo xx, ese modelo autónomo de administración y gestión del $\mathrm{CN}$, apenas muy puntualmente controlado y fiscalizado por el gobierno. Un ejemplo paradigmático se encuentra en los procesos de selección del profesorado: «el Estado portugués se limitó a ejecutar lo que la ley obligaba abriendo concursos públicos» evaluados por un jurado formado por profesores del Conservatorio que controlaba, por tanto, todo el proceso de admisión. A pesar de esto, «ha sido desde siempre permitida al director del Conservatório la contratación de profesores, para cualquier categoría, sin concurso público» con la única condición de que "quedase demostrada la aptitud del candidato». ${ }^{11}$

En cuanto a la financiación del CN, el modelo heredado de la Monarquía y seguido por el gobierno republicano suponía que los sueldos de los profesores y trabajadores no docentes eran liquidados directamente por el Estado. A través de su presupuesto general anual, el Gobierno central atribuía además otra subvención destinada a la «adquisición de instrumentos y sus reparaciones, cuerdas para instrumentos, libros para

\footnotetext{
${ }^{8}$ Designación adoptada en 1910 para el hasta entonces denominado Real Conservatorio de Lisboa. La generalidad de referencias en esa investigación se refiere a su sección de música, que desde 1901 tenía una gestión independiente de la sección de teatro.

${ }^{9}$ Caspurro afirma que, desde la fundación del CN, «la música entra definitivamente — aunque de forma autónoma en el ámbito de las estructuras educativas- en el cuadro del sistema educativo portugués» cf. Maria Helena Ribeiro da Silva Caspurro, «O Conservatório de Música do Porto: das origens à integração no Estado» (Tesis de Máster, Universidade de Coimbra, Faculdade de Letras, 1992), 16 y 17. Ajeno al ámbito de responsabilidad gubernamental, es importante referir la fundación en 1884 de la Real Academia de Amadores de Música, una institución que empezó por ser promotora de conciertos con orquesta propia, pero que algunas décadas más tarde abandonaría esa prioridad para ser sobre todo una escuela de música. Su importante legado y actividad persisten hasta el presente. Según Nery, esta ha «contribuido de forma decisiva para una expansión del espectro de la formación musical profesional, tanto en cantidad como en calidad. $C f$. Rui Vieira Nery, Os sons da República (Lisboa: Imprensa Nacional Casa da Moeda, 2015), 32.

${ }^{10}$ Fernandes, Ó y Ferreira: Estudo de Avaliação do ensino artístico, 244.

${ }^{11}$ Fernandes, Ó y Ferreira: Estudo de Avaliação do ensino artístico, 247.
} 
las clases, mantenimiento de pianos, gas, agua ${ }^{12} \mathrm{y}$ otros gastos corrientes. La investigación y comparación de las subvenciones atribuidas entre 1909 y 1926 —que incluyen, por tanto, la transición monarquía-república y todo el período republicano - nos permite observar que el valor efectivo de la subvención anual se mantuvo constante. ${ }^{13}$

La legislación oficial relacionada con la enseñanza musical en Portugal entre 1910 y 1926 presupone, una vez más, el análisis del caso concreto del CN, el núcleo fundamental de las políticas educativas del sector. Llegada la República en 1910, el funcionamiento y el organigrama se basaba fundamentalmente en la legislación que resulta de la reforma del año 1901, con la definición de su estructura ${ }^{14}$ y de sus reglas de procedimiento. ${ }^{15}$ Dicha legislación reafirma un cierto estatuto de autonomía del $\mathrm{CN}$ - y por consecuencia de la música - sobre la cual «el legislador lanzó un manto de silencio sobre los métodos y procesos» ${ }^{16}$ en las grandes reformas educativas generales del año 1895. Fernandes et al. afirman incluso que «la documentación perpetúa una especie de temor de las autoridades frente a los poderes culturales, especialmente musicales». ${ }^{17}$

En 1914, ya consolidada la República, el gobierno ratifica la referida autonomía del $\mathrm{CN}$ con el decreto n. $625^{18}$ recalcando que

Es de gran conveniencia conceder autonomía administrativa a los establecimientos que, por el grado de cultura y formación que ofrecen, sean dignos de serles confiado su propio gobierno educati-

\footnotetext{
${ }^{12}$ Secretaria-Geral do Ministério da Educação e Ciência, «Conservatório Nacional: Libros de registro de gastos e ingresos 1909-1925», A01, Archivo de la Secretaria-Geral do Ministério da Educação e Ciência.

${ }^{13}$ De hecho, era común hacer un ajuste posterior del subsidio, para hacerlo corresponder con los gastos factuales del CN. En 1909 se atribuyeron 898.000 réis (moneda oficial monárquica) y los gastos se situaban en los 74.830 réis mensuales. Entre 1910 y 1917 se mantiene prácticamente inalterada la subvención anual de 1.280 escudos (moneda oficial republicana), valor efectivo un poco superior a la subvención atribuida antes de 1910 (tasa de cambio 1000 réis = 1 escudo). Entre 1918 y 1926 la subvención aumentó gradualmente $(1918=1.350$ escudos; $1919=1.999,92$ escudos; $1920=3.000$ escudos; $1922=19.887,63 €)$, una variación justificable y equivalente con la devaluación de la moneda portuguesa en los años posteriores a la Primera Guerra Mundial. Cf. Secretaria-Geral do Ministério da Educação e Ciência, «Conservatório.Nacional» .

${ }^{14}$ Decreto de 24.10.1901 publicado en el Diário do Governo n. 242 de 26.10.1901.

${ }^{15}$ Decreto de 22.11.1901 publicado en el Diário do Governo n. 267 de 26.11.1901.

${ }^{16}$ Fernandes, Ó y Ferreira: Estudo de Avaliação do ensino artístico, 244.

${ }^{17}$ Fernandes, Ó y Ferreira: Estudo de Avaliação do ensino artístico, 244.

${ }^{18}$ Decreto n. 625 publicado en el Diário do Governo n. 109 de 04.07.1914. «https://dre.pt/application/ file/479348”
} 
vo y económico. [...] Entre esos establecimientos deben incluirse la Escola de Arte de Representar y la de Música [del Conservatorio]. ${ }^{19}$

Exceptuando algunos decretos — por ejemplo, sobre los concursos para admisión de alumnos y profesores u otros temas de gestión corriente- hubo que esperar hasta 1919 para que el gobierno republicano decretase una verdadera reforma en el $\mathrm{CN}$, ordenando su remodelación ${ }^{20}$ y estipulando nuevas reglas de procedimiento. ${ }^{21}$ Dicha reforma fue el resultado del trabajo de una comisión nombrada en 1918 por el gobierno para remodelar la enseñanza artística musical, comisión en la que se incluían destacadas personalidades del sector de la música clásica. ${ }^{22}$ Branco calificó esta reforma de «notable» y destacó la «modernización de los programas y los métodos pedagógicos» además de que se hubieran creado «medios para obtener una cultura menos rudimentaria que lo habitual entre los músicos portugueses». ${ }^{23}$

Conviene mencionar también los ajustes y alteraciones al programa curricular del CN en $1923^{24}$ y $1925,{ }^{25}$ que vienen además al encuentro de las críticas del compositor Luís de Freitas Branco $^{26}$ sobre la necesidad de una «profunda remodelación» ${ }^{27}$ en dichos programas.

\footnotetext{
${ }^{19}$ La misma legislación refiere también que «la administración económica [...] será confiada [...] a un consejo administrativo constituido por el respectivo director, que será el presidente, y por dos miembros elegidos por el cuerpo docente» y que el gobierno "podrá inspeccionar y fiscalizar la administración [...] siempre que lo considere necesario o conveniente».

${ }^{20}$ Decreto n. 5546 de 09.05.1919 publicado en el Diário do Governo n. 97 de 09.05.1919. «https://dre. pt/application/file/204092». Rectificado en el Diário do Governo n. 193 de 23.09.1919. https://dre.pt/ application/file/359074.

${ }^{21}$ Decreto n. 6129 de 25.09.1919 publicado en el Diário do Governo n. 195 de 25.09.1919. «https://dre. pt/application/file/359049»

${ }^{22}$ Sus miembros eran el político António Arroio (presidente), el pianista y compositor José Viana da Mota, el pianista Alexandre Rey Colaço, el musicologo Michel'angelo Lambertini y el compositor Luís de Freitas Branco (se refieren en este caso solo las actividades profesionales por las cuales estas personalidades son más conocidas).

${ }^{23}$ João de Freitas Branco, História da Música Portuguesa, 4ạ. ed. (Lisboa: Biblioteca da História, 1959), 296.

${ }^{24}$ En Diário do Governo n. 3 de 04.01.1923. https://dre.pt/application/file/240112.

${ }^{25}$ En Diário do Governo n. 39 de 19.02.1925. https://dre.pt/application/file/436940.

${ }^{26}$ Curiosamente, uno de los mentores de la reforma de 1919 y subdirector del mismo Conservatorio entre 1919 y 1924.

${ }^{27}$ Citado en Carlos Alberto Faísca Fernandes Gomes, «Discursos sobre a especificidade do ensino artístico: a sua representação histórica nos séculos XIX e XX» (Tesis de Máster, Universidade de Lisboa, Faculdade de Psicologia e Ciências da Educação, 2002), 130.
} 


\section{EL CONSERVATÓRIO NACIONAL: DE CENTRALIZADOR A MOTOR DE LA DESCENTRALIZACIÓN}

Ya desde 1841, apenas seis años después de su creación, queda definida, a respecto a la posible fundación de filiales del Conservatorio Nacional $(\mathrm{CN})$ en otras ciudades portuguesas, "la relación entre la institución madre y las delegaciones». Esto representó otra "concesión sin precedentes [...] en el sistema público de educación portugués». ${ }^{28}$ De todos modos, tendríamos que avanzar varias décadas hasta poder percibir las primeras señales explícitas sobre la expansión institucional de la música en el sistema educativo público. El $\mathrm{CN}$ fue, durante varias décadas, el aglutinador de la acción del poder en el ámbito de la educación para la música clásica. Mirar hacia la «legislación como umbral de la racionalidad del Estado y de la relación tutelar», significa concentrarse casi exclusivamente en la existencia del Conservatorio, la «única institución mantenida en esta área por el Estado». ${ }^{29}$

La gran excepción es seguramente la Universidad de Coimbra —la más antigua de Portugal - por haber albergado una enseñanza oficial de música desde $1544 .{ }^{30}$ Tradicionalmente integrada en su Capilla desde hacía varios siglos y «en función de los tiempos de cambio [...] después de la implantación de la República», se debatió «el reconocimiento de su valor formativo y el rechazo por su afinidad con la Capilla de la Universidad». ${ }^{31}$ Era un momento particularmente crítico para la institución, sobre todo cuando entraron en competencia las recién creadas Universidades de Lisboa y Oporto en 1911.32 Finalmente se opta por la re-estructuración de la enseñanza musical, transformándola en asignatura de Historia de la Música y asegurando su persistencia en la Universidad de Coimbra hasta el presente. Un elemento crucial en esta decisión, fue también la

\footnotetext{
${ }^{28}$ Fernandes, Ó y Ferreira: Estudo de Avaliação do ensino artístico, 245.

${ }^{29}$ Ana Paz, «Ensino da Música em Portugal (1868-1930): uma história de pedagogia e do imaginário musical» (Tesis de Doctorado, Universidade de Lisboa, Instituto de Educação, 2014), 174.

${ }^{30}$ Monteiro afirma que la música formaba parte del plan curricular de universidad portuguesa «probablemente desde su fundación» en 1290 o por lo menos «de forma comprobada» desde 1323. Cf. Maria do Amparo Carvas Monteiro, "A Universidade de Coimbra e as Ciências Musicais», Revista Brasil-Europa 111 (2008): 2.

${ }^{31}$ Monteiro, «A Universidade de Coimbra e as Ciências Musicais», 2.

${ }^{32}$ Decreto-lei de 22.03.1911 publicado en el Diário do Governo de 24.03.1911. https://dre.pt/application/dir/pdfgratis/1911/03/06800.pdf.
} 
ley n. 861 de 1919 que oficializaba «la práctica musical de los orfeones académicos y la investigación y estudio de las canciones nacionales y su adaptación a la enseñanza».33

Con la llegada de la República en 1910, se intensificaron las voces que reivindicaban la creación de escuelas de música oficiales fuera de Lisboa, una idea seguramente compartida por muchos, pero que se revelaría difícil de concretar inmediatamente por motivos económicos y organizativos. En realidad, la descentralización educativa en el ámbito musical acabaría por tener en el mismo $\mathrm{CN}$ uno de sus actores principales. Lo que en un principio puede parecer una contradicción - ¿el centralizador Conservatorio de Lisboa promoviendo la descentralización? - se puede justificar fácilmente con el rápido crecimiento del mismo $\mathrm{CN}$ en estos años.

Tabla 1. Adaptada de Fernandes, Ó y Ferreira:

Estudo de Avaliação do ensino artístico, 254.

\begin{tabular}{|c|c|c|}
\hline Año escolar & Alumnos internos & Alumnos externos \\
\hline $1885-1886$ & 231 & 489 \\
\hline $1900-1901$ & 331 & 580 \\
\hline $1915-1916$ & 774 & 1231 \\
\hline $1930-1931$ & 1162 & 974 \\
\hline $1945-1946$ & 261 & 611 \\
\hline
\end{tabular}

La tabla anterior describe la evolución de las matrículas de alumnos internos y externos ${ }^{34}$ del $\mathrm{CN}$ durante la Primera República y algunas décadas anteriores y siguientes, con la finalidad de clarificar aún más las tendencias de esa misma evolución. En los primeros años del período republicano «la demanda de algún tipo de formación musical no paraba de aumen$\operatorname{tar}^{35}$ - tanto en alumnos internos como externos- y urgía una solución para la creciente saturación pedagógica, logística y organizativa del $\mathrm{CN}$.

\footnotetext{
${ }^{33}$ Lei n. 861 publicada en el Diário do Governo de 27.08.1919. «https://dre.pt/application/file/360408» ${ }^{34}$ Alumnos de profesores particulares autorizados por el CN, institución que acogía además sus exámenes periódicos.

${ }^{35}$ Paz, «Ensino da Música em Portugal (1868-1930)», 5.
} 
Por un lado, a mediados de 1920 se tomó la «decisión política de comprimir y distribuir la educación musical en otras vías» llevada a cabo a mediados de 1920 para a disociar gradualmente la «formación de amateurs y no profesionales ${ }^{36}$ de otra formación más especializada en el CN. En los Decretos 5546 y 6129, respectivamente de 9 de mayo y 25 de septiembre de 1919, se toman medidas, por ejemplo, en el caso muy concreto de la capital portuguesa con las que se pretendía «aliviar el Conservatorio de las clases de solfeo» pasando estas a ser «impartidas por (otra) escuela primaria de música». Fernandes et al. destacan, sin embargo, que «en este intento de difundir la oferta no estaba implícita cualquier idea de democratizar y de masificar la enseñanza musical», ${ }^{37}$ dando a entender que las medidas se basaban más bien en una necesidad relacionada con la referida saturación del $\mathrm{CN}$.

Por otro lado, el «idealismo educacional de la legislación republicana» parecía favorecer, al menos teóricamente, la indispensable expansión de la formación musical a otras ciudades portuguesas. En el caso de la enseñanza artística especializada, la fundación de un Conservatorio en Oporto - lograda por iniciativa municipal — fue seguramente el acontecimiento más relevante de la época. La prioridad de los políticos republicanos era, por encima de todo, «organizar en las capitales de distrito y otros centros de población, escuelas primarias de música con formación de solfeo orado y cantado, teoría elemental de música y canto coral». ${ }^{38}$ La imposibilidad de financiar este proyecto desde el Estado asignaba una vez más a las instituciones regionales y municipales la difícil tarea de «encontrar los ingresos necesarios para apoyar el funcionamiento de un centro escolar de esta naturaleza».39 Según describe Ana Paz «durante la primera República portuguesa [...] se tomaron importantes medidas legales [...] para la implementación de una asignatura formalmente designada por Música» en los planos curriculares de la enseñanza pública, medidas esas que fueron «implementadas» a partir de 1916 y «posterior-

\footnotetext{
${ }^{36} \mathrm{Paz}$, «nsino da Música em Portugal (1868-1830», 5.

${ }^{37}$ Fernandes, Ó y Ferreira: Estudo de Avaliação do ensino artístico, 245.

${ }^{38}$ «En algunos niveles [...] la educación musical tenía además de teoría y práctica de canto coral posibles extensiones a una práctica instrumental, por ejemplo el piano» $c f$. Paz, «Ensino da Música em Portugal (1868-1930)», 443.

${ }^{39}$ Fernandes, Ó y Ferreira: Estudo de Avaliação do ensino artístico, 245.
} 
mente desarrolladas (pero no necesariamente ampliadas) por la política autoritaria del Estado Novo» ${ }^{40}$.

A pesar de todo, debemos reconocer que la Primera República, cuya acción sería decisiva en la reestructuración del sistema educativo en Portugal en la mayoría de las áreas restantes, se acordó tarde de la formación profesional especializada de los músicos y no pudo llegar a tiempo de revertir el legado de inoperancia heredado del Liberalismo. ${ }^{41}$

Si Portugal ya padecía de fragilidades en cuestión de tradición musical y su correspondiente educación, podemos observar que el poder político republicano no ha podido compensar o contrariar esa tendencia. Como podremos verificar más adelante en esta investigación, sólo varias décadas después se dieron pasos concretos y sólidos en la creación de escuelas de música en otras ciudades del país.

\section{EL CANTO CORAL Y LA REPÚBLICA}

A los ideales de civilización, patriotismo, democracia y libertad, se opone la anarquía, el conservadurismo, el oscurantismo, la inercia y la decadencia. Tal como el canto coral es la bandera de los primeros, se nota su ausencia en los segundos. ${ }^{42}$

El reconocimiento del canto coral como práctica agregadora de valores positivos y modélicos gana aún más protagonismo con el advenimiento de la República y con la subsiguiente implementación de sus ideales. La cualidad educativa y civilizadora del canto coral y «el ideal de transformar su composición heterogénea en una sociedad homogénea, neutral, que sirviese de modelo para la sociedad civil» ${ }^{43}$ se han considerado sumamente oportunos en los años pos-revolución. La población portuguesa padecía de claros déficits de formación y educa-

\footnotetext{
${ }^{40}$ Paz, «Ensino da Música em Portugal (1868-1930)», 443.

${ }^{41}$ Nery, Os sons da República, 34.

${ }^{42}$ António Arroyo, O Canto Coral e a sua Funçao Social (Coimbra: França Amado, 1909), 18. Citado en Barreiros, «A disciplina de Canto Coral no período do Estado Novo», 55 y 56.

${ }^{43}$ Barreiros, «A disciplina de Canto Coral no período do Estado Novo», 57.
} 
ción - una situación aún más alarmante si se compara con la de otros países europeos- y la institucionalización del creciente movimiento orfeónico representó una importante oportunidad de atraer al país a través de la música. Las características asociativa y de convivencia de los grupos corales y la forma en la que estos podían fomentar «los ideales de la humanidad y de la fraternidad», ${ }^{44}$ en paralelo con sus rasgos de ciudadanía, atribuían al canto coral una enorme importancia en el contexto de un nuevo sistema político y de nuevos desafíos en la sociedad portuguesa. ${ }^{45}$

Además, es realmente significativo que «en el establecimiento de un nuevo orden social y político, el canto coral parece haber constituido tanto para la República como (más tarde) para el Estado Novo, una componente inseparable de sus ideales» ${ }^{46}$ : un dato muy importante para una investigación sobre la relación entre la música y el poder.

El canto coral surgió por primera vez en el «plan de estudios portugués [...] en el ámbito de la educación intelectual» en $1870,{ }^{47}$ y muy concretamente en la "enseñanza primaria complementaria» ${ }^{48}$ en $1878 . .^{49}$ De todas formas, no era objeto de especial interés por parte de los alumnos, los profesores o el poder central, limitándose a ser una actividad lúdica complementaria impartida por docentes sin formación musical. En 1906, aparece también en el plan de estudios ${ }^{50}$ del liceo femenino Maria Pia de la capital portuguesa. El canto coral acabaría por aparecer

\footnotetext{
${ }^{44}$ Barreiros, «A disciplina de Canto Coral no período do Estado Novo», 127.

${ }^{45}$ Tomás Borba (1867-1950), importante pedagogo musical y editor responsable de la elaboración de algunos de los principales manuales de canto coral durante la Primera República, afirma en 1912 que «el problema de la canción escolar en el actual momento histórico es ahora de más difícil solución que jamás, por la secularización o neutralidad en materia de religión en el que los gobiernos del régimen ahora adoptado quieren mantener la educación». Cf. Tomás Borba, Revista de Educação Geral e Technica I, no. 3 (1992): 237, citado en Barreiros, «A disciplina de Canto Coral no período do Estado Novo», 104.

${ }^{46}$ Barreiros, «A disciplina de Canto Coral no período do Estado Novo», 127.

${ }^{47}$ Decreto de 16.08.1870.

${ }^{48}$ Fernando José Monteiro da Costa, «Canto Coral, escola de higienização», Revista da Faculdade de Letras - História III, no 11 (2010): 237.

${ }^{49}$ Ley de 02.05.1878.

${ }^{50}$ Decreto de 31.01.1906, en el que se afirma que «la educación de una madre de familia no se puede considerar completa» sin un conjunto de asignaturas, entre las cuales figuran «la música, la moral, la higiene, la culinaria, la pedagogía». Cf. Costa, "Canto Coral, escola de higienização», 238. Los liceos masculinos no impartían estas asignaturas.
} 
en la legislación republicana apenas en $1918,{ }^{51}$ con su introducción oficial como asignatura propia y obligatoria en la educación secundaria: en ella quedaba definida su función «estética, moralizadora y nacionalista» y el objetivo de que con el canto coral se proporcionase a los alumnos «una gran lección de moral, belleza, civismo y solidaridad».52 En 1921 se aprueba un nuevo reglamento para la educación secundaria ${ }^{53}$ que no altera sustancialmente lo anterior pero introduce algunas novedades como, por ejemplo, la indicación de que «la enseñanza de las bases de musicales debe formar parte de las clases de canto coral, cuando este sea impartido con procesos modernos». ${ }^{54}$. Se puede identificar esta señal como primer precedente específico de una futura asignatura de música, solfeo o formación musical.

En general, «la enseñanza coral se había establecido con efectividad, tanto en escuelas como en asociaciones corales de todos los espectros de la sociedad: ejército, asociaciones académicas y otros gremios», e «incluso se practicaba de distintas formas en las élites». ${ }^{55}$ Por otro lado, «se nota por primera vez en la historia de la prensa sobre educación (musical), una gran inversión en el tema del canto coral» cuando algunas de las principales publicaciones de la época en este ámbito ${ }^{56}$ insistieron en «la defensa del canto escolar en momentos de decisión política». ${ }^{57}$

Pero también son conocidas las vulnerabilidades de su institucionalización. En 1923 se lamentaba, por ejemplo, «la falta de un programa diseñado correctamente ${ }^{58}$ y la incompetencia de los que deberían elaborarlo. Al mismo tiempo, se denunciaba que los profesores de canto

\footnotetext{
${ }^{51}$ Decreto n. 4650 en Diário do Governo n. 157 de 14.07.1918. <https://dre.pt/application/file/244447> y Decreto n. 4799 en Diário do Governo n. 198 de 08.09.1918. <https://dre.pt/application/file/169541>.

${ }^{52}$ En Diário do Governo n. 123 de 18.06.1918. <https://dre.pt/application/file/169541>.

${ }_{53}^{3}$ Decreto n. 7558 en Diário do Governo n. 123 de de 18.06.1921. <https://dre.pt/application/file/510738>.

${ }^{54}$ Decreto n. 7558 en Diário do Governo n. 123 de de 18.06.1921. <https://dre.pt/application/file/510738>.

${ }_{55}^{5}$ Paz, «Ensino da Música em Portugal (1868-1930)», 448.

${ }^{56}$ Ana Paz enumera A arte musical (1899-1915), el Boletim da Associação de Classe dos Músicos o Eco musical (1911-1917), además de Vida musical (1923), Santa Cecília (1923), Música (1924-1925) o, en 1930, Arte musical. Cf. Paz, «Ensino da Música em Portugal (1868-1930)», 444.

${ }^{57}$ Paz, «Ensino da Música em Portugal (1868-1930)», 444.

${ }^{58}$ Real Costa: O Ensino da Música em Portugal: o que ele é o que devia ser, 1923, Cf. Barreiros, «A disciplina de Canto Coral no período do Estado Novo», 59.
} 
coral sufrían condiciones precarias por ser los «únicos que no formaban parte del cuadro de los respectivos centros de enseñanza», y que recibían "sueldos inferiores a los trabajadores no docentes». ${ }^{59}$ De igual modo, era considerado perjudicial el hecho de que las clasificaciones obtenidas en la asignatura de canto coral no tuviesen influencia en las calificaciones globales del alumno y había un claro malestar por los contratos anuales de los profesores de canto coral y por los criterios seguidos en su nombramiento y selección.

A la luz de toda la información reunida y analizada, es posible confirmar que «como en otros campos de la política educativa» durante la Primera República, «los medios se quedaron muy por debajo de los fines» en su objetivo de garantizar «el reconocimiento y la aplicación efectiva » ${ }^{60}$ del canto coral en el sistema educativo portugués. Hacer alusión a «la república que prometía mucho y cumplía apenas en parte» será probablemente adecuado también en el caso del canto coral. Asimismo, en el curso de esta investigación podremos verificar que su institucionalización y politización superará los limites cronológicos de la Primera República.

\section{EL CONSERVATORIO DE MÚSICA DO PORTO Y SU RELACIÓN CON EL (LOS) PODER(ES)}

La ciudad portuguesa de Oporto, segunda más importante del país, disponía a finales del siglo xIX de una importante actividad en el dominio de la música clásica. Sorprendentemente o no, esa actividad no estaba respaldada por ninguna institución de educación musical especializada. La formación de destacados músicos locales, como por ejemplo el pianista y director de orquesta Raimundo de Macedo (1889-1931), los pianistas y compositores Óscar da Silva (1870-1958), Luís Costa (18791960) y Hernâni Torres (1881-1939), el violinista y director Bernardo Valentim Moreira de Sá (1853-1924) y la violonchelista Guilhermina Suggia (1885-1950) —algunos de ellos con significativas carreras también en el extranjero- sólo resultó de la vigorosa y prolífera dinámica de clases particulares que emanaba en la ciudad. ${ }^{61}$

\footnotetext{
${ }^{59}$ Armando Leça, «O canto coral nos liceus», Vida Musical 2 (1923): 12.

${ }^{60}$ Barreiros, «A disciplina de Canto Coral no período do Estado Novo», 127.

${ }^{61}$ «La enseñanza impartida en círculos particulares era en efecto valiosa pero una vez que no contemplaba la existencia de un cuadro curricular y sistematizado [...] acababa por ser reconocida apenas
} 
Los primeros antecedentes del Conservatorio no pasaron de efímeras existencias de centros de enseñanza como la Escola Popular de Canto (1855-1859) o el Instituto Musical (1863-1864), ambos con apoyo del poder político local y ubicados físicamente en el edificio del ayuntamiento. La receptividad de los alumnos a ambos proyectos fue entusiasta y numerosa. En el caso de la Escola Popular de Canto se inauguró «la actividad escolar con 60 alumnos, número que en algunas semanas superaba ya los 200 para, meses después, ascender a más de $300 »{ }^{62}$ De carácter privado, se pueden referir escuelas más pequeñas, impulsadas por órdenes religiosas o asociaciones socio-profesionales, pero sobre todo la Academia de Música do Palácio de Cristal (1866-1868) que, a pesar de haber quedado "al margen de los auspicios oficiales de la municipalidad», se puede considerar como el último intento del «movimiento de institucionalización de la educación pública de música artística iniciado [...] con la Escola Popular de Canto ${ }^{63}$ algunos años antes.

La urgencia y la necesidad de tener un Conservatorio comparable al de la capital era una evidencia para cualquiera que conociese la realidad musical de la ciudad de Oporto, y ya «desde mediados del siglo XIX, se verifican a menudo apelaciones a las autoridades municipales por parte de los músicos y críticos portuenses ${ }^{64}$ : «no hay nada que pueda disculpar la no existencia de una escuela oficial en Oporto, segunda ciudad del reino, que por su importancia material tendría todo el derecho a la misma», 65 reivindicaba en 1897 el pianista y futuro subdirector del Conservatório de Música do Porto (CMP) Ernesto Maia. El autor consideraba además

inaceptable que el gobierno gaste grandes sumas de dinero en la contratación de músicos profesionales en el extranjero para las compañías de teatro [...] y no atendiese prioritariamente a la formación de sus propios talentos, en especial los que demostraban poder tener éxito en el futuro. ${ }^{66}$

\footnotetext{
al nivel del aficionado». Cf. Caspurro, "O Conservatório de Música do Porto», 30.

${ }^{62}$ João-Heitor Rigaud, O Porto musical no início do séc. Xx (Porto: Meloteca, 2013), 3.

${ }^{63}$ Caspurro, «O Conservatório de Música do Porto», 34.

${ }^{64}$ Caspurro, "O Conservatório de Música do Porto», 17.

${ }^{65}$ Ernesto Maia, «O Ensino Musical - Necessidade da sua Vulgarização», O Primeiro de Janeiro, 29 (1897): 260-263, citado en Caspurro, «O Conservatório de Música do Porto», 49.

${ }^{66}$ Caspurro, "O Conservatório de Música do Porto», 49.
} 
El «vacío institucional» verificado desde el último tercio del siglo XIX hasta la fundación del CMP en 1917 no se podrá seguramente atribuir a «una actitud pasiva por parte de los músicos portuenses o por parte de sus representantes municipales». Ambos eran "conscientes de los perjuicios de un sistema educativo exclusivamente concebido para dar respuesta a la población de la capital» e incluso en «declaraciones oficiales del Ayuntamiento» era evidente «la incompatibilidad con la orientación política de los gobernantes nacionales en el dominio de la educación oficial de música». ${ }^{67}$

En este sentido, hemos de observar también que no siempre el poder central se demostró indiferente o negativo sobre la situación en Oporto. Resulta muy elocuente a este respecto la siguiente transcripción de un extracto del decreto sobre la reforma del Real Conservatório de Lisboa en 1901:

Un país musical es un país adecuadamente preparado para aceptar todas las lecciones de progreso [...] es intención del gobierno de Su Majestad instituir sucursales del Conservatorio, en lo que se refiere a la educación musical, en los diferentes distritos del reino, empezando por los de Oporto [...] cuando las posibilidades del Tesoro lo permitan. Será un medio práctico de propagar por todo el país el gusto por la música y de fomentar el aumento del número de artistas musicales. ${ }^{68}$

Poniendo énfasis en la condición «cuando las posibilidades del Tesoro lo permitan», y sin que sirva de excusa a la evidente falta de sensibilidad que había demostrado el poder central a lo largo de varias décadas, nos encontramos con el motivo clave de este problema: la ausencia de financiación o, alternativamente, la falta de voluntad en canalizar para el proyecto de creación del CMP el presupuesto necesario. «Ante la pasividad de la política nacional [...] es en sus representantes municipales, o incluso en instituciones privadas, que los músicos portuenses encuentran viabilidad $»^{69}$ para hacer realidad sus aspiraciones. En honor a la verdad, se estaba recurriendo al poder local para lograr un «apoyo que debería

\footnotetext{
${ }^{67}$ Caspurro, «O Conservatório de Música do Porto», 48.

${ }^{68}$ Decreto de 24.10.1901. Cf. Caspurro, «O Conservatório de Música do Porto», 52.

${ }^{69}$ Decreto de 24.10.1901, cf. Caspurro, «O Conservatório de Música do Porto», 17.
} 
ser competencia del Estado» ${ }^{70}$ cuanto más no fuera por una cuestión de equilibrio y justicia entre Lisboa y Oporto.

Teniendo en cuenta ciertas teorías de economía política -muy comunes hoy en día- la existencia de escuelas de arte emanando directamente del Estado y apoyadas por fondos públicos no parece fácilmente justificable. La mayoría de los economistas modernos no admite, en materia de arte, más que la pura y simple iniciativa particular, alegando que el Estado debe abstenerse completamente de todo lo que no corresponda a un interés social inmediato y positivo. ${ }^{71}$

En 1914, animado por «los ideales del joven régimen republicano» y "comprometido con el desarrollo de la educación a escala popular», ${ }^{72} \mathrm{el}$ Ayuntamiento de Oporto incluye una propuesta para la creación del CMP en un amplio plan para la educación pública de la ciudad. Pero apenas en 1917 se verifica la entrada real del tema en la orden del día de los trabajos del ayuntamiento. Con base en el decreto de Hintze Ribeiro anteriormente referido, se crea una comisión «que se encarga de estudiar la organización del Conservatorio [...] para la cual se espera el apoyo económico del Estado, como expresa el decreto de 24 de octubre de 1901 [...] quedando establecido que será pedido al gobierno un subsidio». ${ }^{73}$ Este subsidio tardaba en ser concedido y el ayuntamiento decide finalmente avanzar con sus medios propios ${ }^{74}$ autorizando a mediados de julio de 1917 la apertura de matrículas: «La organización y los estatutos han sido aprobados por el Ayuntamiento el día 31 de diciembre de $1917 \gg^{75}$ y las clases empiezan en enero de 1918 con un total de 373 alumnos.

En todo el proceso, fue muy destacable la acción del poder municipal, que demostró «una especial dedicación por los asuntos

\footnotetext{
${ }^{70}$ Decreto de 24.10.1901, cf. Caspurro, «O Conservatório de Música do Porto», 56.

${ }^{71}$ Michelangelo Lambertini, «Sociedade de Concertos Sinfónicos Portuense (Relatório)», A Arte Musical, 17 (1915): 108 y 109; citado en Caspurro, «O Conservatório de Música do Porto», 55.

${ }^{72}$ Michelangelo Lambertini, «Sociedade de Concertos Sinfónicos Portuense (Relatório)», 53.

${ }^{73}$ Michelangelo Lambertini, «Sociedade de Concertos Sinfónicos Portuense (Relatório)», 57.

${ }^{74}$ Arquivo Histórico do Conservatório de Música do Porto, manuscrito 268 (1917), 225; citado en Caspurro, «O Conservatório de Música do Porto», 58.

${ }^{75}$ Rui Moreira de Sá e Guerra, Bernardo Valentim Moreira de Sá (1853-1924): um renovador da cultura musical no Porto (Porto: Fundação Eng. António de Almeida, 1997), 81.
} 
de la educación y de cultura artística al hacer posible la creación y manutención del Conservatório [...] no dejando de demostrar también un igual cuidado organizativo rodeándose de los más competentes elementos para la elaboración y efectuación de un plan educativo basado en principios pedagógicos y artísticos sólidos. ${ }^{76}$

Entre otras cosas, el Ayuntamiento ha asumido la responsabilidad de analizar y aprobar el cuerpo docente y los cargos directivos, después de que estos les hayan sido propuestos por una comisión compuesta por representantes instituciones musicales de la ciudad con elevado prestigio. De esta forma, «a los concejales portuenses no les podía quedar cualquier tipo de duda sobre la credibilidad de la lista presentada». ${ }^{77}$

Sobre el primer plan de estudios del CMP, elaborado en gran medida por su recién nombrado director, el pianista Bernardo Valentim Moreira de Sá, escribió el director del CN de Lisboa José Viana da Mota:

Ha denotado un talento de organización fuera de lo común. [...] Con su cultura universal y su extraordinario método de trabajo, ha organizado un programa de estudios tan correcto para su querida escuela que, teniendo yo que reformar los programas del Conservatorio de Lisboa en 1919, me ha bastado limitarme al plan por él redactado. ${ }^{78}$

Esa reforma del CN en 1919, coincide de hecho con una reestructuración al nivel del plan de estudios en el CMP, aunque no sea posible identificar una relación entre ambas. El CMP funcionaba con total autonomía y esta reestructuración en 1919 fue de

exclusiva iniciativa del consejo escolar del Conservatorio de Oporto y del Ayuntamiento del que dependía, manteniéndose ajeno a cualquier orden del entonces Ministério da Instrução Pública, que a través de su Direcção Geral de Belas Artes se hacía cargo del funcionamiento del Conservatório Nacional. ${ }^{79}$

\footnotetext{
${ }^{76}$ Caspurro, «O Conservatório de Música do Porto», 72.

${ }^{77}$ Caspurro, «O Conservatório de Música do Porto», 59.

${ }^{78}$ José Viana da Mota y Oliva Guerra: In Memoriam (Lisboa: Ramos, Afonso \& Moita, 1952), 13.

${ }^{79}$ Caspurro, «O Conservatório de Música do Porto», 73.
} 
Esa autonomía se mantendría en su generalidad hasta 1972, año en el que el gobierno decreta que el CMP pasa a quedar bajo la tutela del Estado. ${ }^{80}$ De todos modos, en 1924 es decretado el reconocimiento y validad oficial de sus exámenes y diplomas, ${ }^{81}$ ello se entiende como "la primera manifestación oficial de reconocimiento y credibilidad» sobre la actividad del mismo y sobre la «acción municipal por la que ha sido impulsado», pero también acabaría por ser el primer paso hacia el «derecho de soberanía del entonces Ministério da Instrução Pública» ${ }^{82}$ con relación al CMP. Este reconocimiento del plan curricular sería decretado bajo la condición de que este se correspondiera con el practicado en el CN. ${ }^{83}$ Por otro lado, y aunque quedase "preservada la dependencia administrativa y ejecutiva» del Ayuntamiento, queda también «reservado al Ministério da Instrução Pública el derecho - hasta entonces perteneciente al órgano municipal- de fiscalización pedagógica y de reglamentación en la concesión de plazas para el cuerpo docente». ${ }^{84}$

\section{CONCLUSIÓN}

El caso y la historia concreta del Conservatorio de Música do Porto acaba por ser un ejemplo muy descriptivo de las relaciones entre el poder y la música clásica durante el la Primera República: se verifican varios avances y retrocesos sobre su fundación, que acaba por realizarse por iniciativa y mérito del poder municipal y por la insistencia de la iniciativa privada. El poder central republicano, de quien se esperaba o exigía por lo menos un mínimo de responsabilidad, no participa ni apoya —más allá de las fútiles declaraciones de intenciones-en tan importante acontecimiento.

\footnotetext{
${ }^{80}$ Decreto n. 519 publicado en el Diário do Governo n. 289, Série I, de 14.12.1972. <https://dre.pt/ application/file/685320>.

${ }^{81}$ Decreto n. 104 publicado en el Diário do Governo n. 289, Série I, de 31.12.1924. <https://dre.pt/ application/file/562734>.

${ }^{82}$ Caspurro, «O Conservatório de Música do Porto», 78 y 79.

${ }^{83}$ Entre 1924 y 1972, «se considera legítimo el ajuste legislativo del Conservatório de Música do Porto en el contexto de las reformas pedagógicas efectuadas por el Conservatório Nacional, porque es con relación a este que en última instancia es regulada estatutariamente la respectiva oficialización de la enseñanza». $C f$. Caspurro, «O Conservatório de Música do Porto», 80.

${ }^{84}$ Caspurro, «O Conservatório de Música do Porto», 80.
} 
En su generalidad, la Primera República queda efectivamente en la historia como un período de grandes proyectos, intenciones, promesas y expectativas, que en larga medida y desafortunadamente para el medio musical portugués no se hicieron realidad. La multiplicidad de poderes no parece haber tenido por resultado en una acumulación de soluciones; además, cabe destacar que la ya referida autonomía y exclusividad del sector musical propició tanto la eficiencia y aligeramiento de procesos como también impidió un cambio de paradigma en el estatuto marginal o no prioritario de la música en el contexto de las políticas educativas.

\section{Nota sobre el autor}

Bruno Borralhinho es miembro de la Orquesta Filarmónica de Dresden y Director Artístico del Ensemble Mediterrain, que fundó en 2002. Es licenciado en Música por la Universität der Künste de Berlín, institución en la que ha obtenido posteiormente un Postgrado (solista). Concluyó el Máster en Gestión Cultural por la Universitat Oberta de Catalunya y trabaja actualmente en su investigación y tesis de Doctorado con el título Poder y Música Clásica en el Portugal del Siglo xx en la Universidad Carlos III de Madrid.

En 2011 fue uno de los participantes del Atelier for Young Festival Managers organizado por la European Festivals Association (Izmir, Turquia) y desde entonces ha participado en varias conferencias académicas.

Como violonchelista, se presentó en las principales salas de concierto por toda Europa, Rusia, Estados Unidos, Canadá, Corea del Sur, Japón, China y Sudamérica y trabajó con distinguidos maestros como Claudio Abbado, Daniel Barenboim, Kur Masur, Herbert Blomstedt, Christoph Eschenbach, Paavo Järvi, Andris Nelsons, entre otros. Su diversificada actividad artística incluye también la dirección de orquesta: se presentó como maestro al frente de varias orquestas en Portugal, Alemania y República Checa. Fue premiado en diversos concursos, entre los cuales destaca el Prémio Jovens Músicos de la radio-televisión estatal portuguesa. La divulgación de la música portuguesa ha sido desde siempre una de sus prioridades, destacando sus grabaciones Página Esquecida (DreyerGaido, 2009) y Portuguese Music for Cello and Orchestra (Naxos, 2016), ambas merecedoras de las mejores críticas a nivel nacional e internacional. 


\section{REFERENCIAS}

Barreiros, Maria José Conde Artiaga. «A disciplina de Canto Coral no período do Estado Novo». Tesis de Máster, Universidade Nova de Lisboa, Faculdade de Ciências Sociais e Humanas, 1999.

Branco, João de Freitas. História da Música Portuguesa, 4. ed. Lisboa: Biblioteca da História, 1959.

Caspurro, Maria Helena Ribeiro da Silva. «O Conservatório de Música do Porto: das origens à integração no Estado». Tesis de Máster, Universidade de Coimbra, Faculdade de Letras, 1992.

Costa, Fernando José Monteiro da. "Canto Coral, escola de higienização». Revista da Faculdade de Letras - História III, no. 11 (2010): 237-238.

Fernandes, Domingos, Jorge Ramos do Ó y Mário Boto Ferreira (coord.). Estudo de Avaliação do ensino artístico. Lisboa: Direcção Geral de Formação Vocacional do Ministério da Educação e Faculdade de Psicologia e de Ciências da Educação da Universidade de Lisboa, 2007.

Gomes, Carlos Alberto Faísca Fernandes. «Discursos sobre a especificidade do ensino artístico: a sua representação histórica nos séculos XIX e XX». Tesis de Máster, Universidade de Lisboa, Faculdade de Psicologia e Ciências da Educação, 2002.

Guerra, Rui Moreira de Sá e. Bernardo Valentim Moreira de Sá (1853-1924): um renovador da cultura musical no Porto. Porto: Fundação Eng. António de Almeida, 1997.

Monteiro, Maria do Amparo Carvas. «A Universidade de Coimbra e as Ciências Musicais». Revista Brasil-Europa 111 (2008): 2.

MотA, José Viana da, y Oliva Guerra: In Memoriam. Lisboa: Ramos, Afonso \& Moita, 1952.

Nery, Rui Vieira. Os sons da República. Lisboa: Imprensa Nacional Casa da Moeda, 2015.

PAz, Ana. «Ensino da Música em Portugal (1868-1930): uma história de pedagogia e do imaginário musical». Tesis de Doctorado, Universidade de Lisboa, Instituto de Educação, 2014.

Peixinho, Ana Teresa y Clara Almeida Santos (coord.). 1911-2011: Comunicação e Educação Republicanas. Coimbra: Universidade de Coimbra, 2011.

Pintassilgo, Joaquim: "A utopia demopédica da República». En Viva a República! 1910-2010, editado por Luís Farinha, 139. Lisboa: Comissão Nacional para as Comemorações da República, Imprensa Nacional Casa da Moeda, 2010.

Rigaud, João-Heitor. O Porto musical no início do séc. XX. Porto: Meloteca, 2013. 